\title{
Cultura de segurança do paciente na Atenção Primária à Saúde
}

\author{
Patient safety culture in Primary Health Care
}

\section{Como citar este artigo:}

Bohrer JKL, Vasconcelos ACL, Bezerra ALQ, Teixeira CC, Andrade J, Santos PHF, et al. Patient safety culture in Primary Health Care. Rev Rene. 2021;22:e70874. DOI: https://doi.org/10.15253/2175-6783.20212270874

(D) Jéssica Karine Lopes Bohrer ${ }^{1}$

(1D)Ana Catarina Laboissière Vasconcelos ${ }^{1}$

DAna Lúcia Queiroz Bezerra²

(D) Cristiane Chagas Teixeira ${ }^{2}$

(D) Juliane Andrade ${ }^{1}$

(D) Paulo Henrique Fernandes dos Santos ${ }^{1}$

(DThatianny Tanferri de Brito Paranaguá ${ }^{1}$

${ }^{1}$ Universidade de Brasília.

Brasília, DF, Brasil.

${ }^{2}$ Universidade Federal de Goiás.

Goiás, GO, Brasil.

\section{Autor correspondente:}

Thatianny Tanferri de Brito Paranaguá

Faculdade de Medicina e Faculdade de Ciências de Saúde Campos Universidade Darcy Ribeiro s/ $\mathrm{n}$ - Asa Norte CEP: 70910-900. Brasília, DF, Brasil.

E-mail: paranagua@unb.br
EDITOR CHEFE: Ana Fatima Carvalho Fernandes

EDITOR ASSOCIADO: Renan Alves Silva

\section{RESUMO}

Objetivo: avaliar a cultura de segurança do paciente em uma unidade de saúde da Atenção Primária. Métodos: estudo transversal, conduzido com 51 profissionais vinculados à Atenção Primária à Saúde. Dados foram coletados utilizando o instrumento autoaplicável Medical Office Survey on Patient Safety Culture, traduzido, adaptado e validado para uso no Brasil. Foi realizada análise estatística descritiva. Participaram enfermeiros, médicos, Agentes Comunitários de Saúde, dentre outros profissionais. Resultados: as dimensões da cultura de segurança do paciente na Atenção Primária à Saúde apontaram oportunidades de melhoria ou fragilidades do serviço. A avaliação geral em segurança do paciente e a avaliação global da qualidade do cuidado prestado na Atenção Primária à Saúde foram apontados como boa ou razoável. Conclusão: a cultura de segurança na Atenção Primária não identificou dimensões fortes, verificaram-se problemas com equipamentos, realização e disponibilidade dos resultados de exames e troca de informações com centros de imagem/laboratórios da rede de atenção.

Descritores: Atenção Primária à Saúde; Cultura Organizacional; Gestão da Segurança; Segurança do Paciente.

\section{ABSTRACT}

Objective: to evaluate the patient safety culture in a primary care health unit. Methods: cross-sectional study conducted with 51 professionals linked to Primary Health Care. Data were collected using the self-administered instrument Medical Office Survey on Patient Safety Culture, translated, adapted and validated for use in Brazil. Descriptive statistical analysis was performed. Nurses, physicians, Community Health Agents, among other professionals participated. Results: the dimensions of the patient safety culture in Primary Health Care indicated opportunities for improvement or weaknesses in the service. The general assessment of patient safety and the global assessment of the quality of care provided in Primary Health Care were appointed as good or fair. Conclusion: the safety culture in Primary Care did not identify strong dimensions; there were problems with equipment, performance and availability of test results and exchange of information with imaging centers/laboratories in the care network.

Descriptors: Primary Health Care; Organizational Culture; Safety Management; Patient Safety. 


\section{Introdução}

A segurança do paciente consiste em um conjunto de ações organizadas que influencia culturas, processos, comportamentos, tecnologias e ambientes da assistência à saúde de forma a reduzir os riscos assistenciais, a ocorrência de danos evitáveis e o seu impacto para paciente, profissional e organização. 0 cuidado primário seguro tem sido considerado prioridade global, sendo incentivada a adoção de estratégias pela Organização Mundial de Saúde ${ }^{(1)}$. A preocupação com a segurança na Atenção Primária à Saúde permeia as práticas assistenciais, especialmente, pelo fato de o usuário não estar sob supervisão dos profissionais a todo momento, como ocorre quando está fora da unidade de saúde, o que dificulta a identificação dos incidentes ${ }^{(2)}$.

Na França, no ano de 2013, incidentes advindos do cuidado prestado na Atenção Primária à Saúde ocorreram, em média, uma vez a cada dois dias, sendo que 2,0\% deles foram associados à possibilidade definitiva de dano e sua ocorrência foi três vezes mais frequentemente relacionada com o gerenciamento dos cuidados assistenciais do que com a competência dos profissionais ${ }^{(3)}$. Na Inglaterra, 58,0\% dos incidentes relatados resultaram em dano ao paciente, dos quais $17,0 \%$ foram graves, resultando em internação hospitalar, lesão permanente ou morte ${ }^{(4)}$. Em países de baixa ou média renda, os incidentes podem atingir entre $25,0 \%$ a $40,0 \%$ dos cuidados primários prestados, sendo que $80,0 \%$ destes são considerados evitáveis ${ }^{(5)}$. No Brasil, no ano de 2018 a análise de 105 notificações verificou dano em $37 \%$ dos casos, incluindo dois óbitos $^{(6)}$.

A ocorrência de incidentes está diretamente relacionados com o processo clínico, medicamentos, tratamento, diagnóstico e gestão do cuidado do paciente $^{(3)}$. No Brasil, estudo apontou como incidentes predominantes àqueles associados aos erros de tratamento, de manejo do prontuário, de comunicação e de registros e agendamento ${ }^{(6)}$. A fim de melhorar esse cenário, em âmbito mundial, e promover melhorias na prestação de cuidado, há um crescente reconhecimento sobre a necessidade de desenvolver a cultura de segurança do paciente. Uma forte cultura de segurança é fundamental para qualquer esforço sustentável em direção à segurança do cuidado; apesar de as políticas e intervenções legislativas poderem fornecer um ambiente propício para o seu desenvolvimento, a cultura de segurança precisa se infiltrar nas atitudes, crenças, valores, habilidades e práticas de saúde ${ }^{(1)}$.

Diante das inúmeras barreiras e múltiplos desafios que necessitam ser enfrentados para melhorar a ocorrência de incidentes na atenção primária( ${ }^{(7)}$, a investigação da cultura de segurança pode auxiliar no reconhecimento de falhas sistêmicas e fomentar mudanças no processo de trabalho. No Brasil, o fortalecimento da cultura nos cenários de atenção à saúde é tema do Programa Nacional de Segurança do Paciente, o qual tem promovido a mobilização de organizações e profissionais de saúde e influenciado a redução de óbitos relacionados com o cuidado inseguro ${ }^{(8)}$.

Diante do exposto e considerando que os incidentes que acontecem na Atenção Primária à Saúde, também, apresentam potencial para danos graves, o presente estudo se justifica por possibilitar o levantamento de indicadores relacionados com as fortalezas e fragilidades do processo de trabalho associadas à cultura de segurança do paciente que podem subsidiar a instalação de processo de melhoria do ambiente assistencial, bem como consolidar o conhecimento sobre a temática, ainda, considerada lacuna científica. Este estudo, portanto, objetivou avaliar a cultura de segurança do paciente em uma unidade de saúde da Atenção Primária.

\section{Métodos}

Trata-se de estudo transversal, desenvolvido em uma unidade de saúde da atenção primária, situada na Regional Leste do Distrito Federal, Brasil. A população do estudo foi composta de 90 profissionais vinculados a seis equipes de saúde da família, uma equipe parametrizada e um Núcleo de Apoio à 
Saúde da Família, sendo nove médicos de família e comunidade, uma médica pediatra, nove enfermeiros, 23 técnicos de enfermagem, cinco odontólogos, cinco técnicos em higiene dental, 25 Agentes Comunitários de Saúde, três técnicos administrativos, três profissionais vinculados às atividades de gestão, uma farmacêutica, uma fonoaudióloga, uma nutricionista, um assistente social, um terapeuta ocupacional e dois auxiliares operacionais dos serviços de patologia clínica e laboratório.

Como critério de inclusão, foi considerado o tempo de atuação superior a 30 dias no serviço. Foram excluídos os profissionais que estavam de férias ou licença por qualquer motivo no período de realização da coleta de dados, e àqueles que não se disponibilizaram a responder ao instrumento após duas tentativas. Não houve perdas relacionadas com o preenchimento incompleto ou incorreto do instrumento; não houve recusas ao convite para participação na pesquisa; 38 profissionais estavam de licença ou férias durante o período de coleta de dados; um profissional foi excluído por esta no serviço há menos de 30 dias. Assim, a população final do estudo foi constituída por 51 profissionais da unidade saúde.

Utilizou-se o instrumento Pesquisa sobre Cultura de Segurança do Paciente para Atenção Primária, versão traduzida do Medical Office Survey on Patient Safety Culture, adaptada para uso no Brasil ${ }^{(9)}$. A validação do instrumento atingiu índice de validade de conteúdo geral de 0,85 e coeficiente alfa de Cronbach de 0,95 . Neste estudo, o alfa obtido foi de 0,90 .

Trata-se de instrumento autoaplicável, composto de nove seções. Sete seções contemplam 52 itens, formatados em escala do tipo Likert: A - Lista de questões sobre segurança do paciente e qualidade; B - Troca de informações com outras instituições; C Trabalhando neste serviço de saúde; D - Comunicação e acompanhamento; E - Apoio de gestores/administradores/líderes; F - Seu serviço de saúde; G - Avaliação global. Dentre esses itens, 38 são agrupados e formam 10 dimensões da cultura de segurança do paciente: Ritmo e pressão no trabalho; Trabalho em equipe; Treinamento da equipe; Processo de trabalho e padronização; Comunicação aberta; Comunicação sobre o erro; Seguimento da assistência ao paciente; Aprendizagem organizacional; Apoio dos gestores na segurança do paciente; Percepção geral da segurança do paciente e qualidade. Os demais itens contemplam perguntas sobre problemas de troca de informações com outros serviços, acesso aos cuidados de saúde, avaliação sobre cinco áreas de qualidade dos cuidados de saúde (centrado no paciente, eficaz, oportuno, eficiente e equitativo) e avaliação geral da segurança do paciente. A Seção H contém perguntas de múltipla escolha sobre o perfil profissional, e a Seção I é composta de questão não obrigatória, dissertativa, sobre segurança do paciente e qualidade assistencial. Foram acrescentadas ao instrumento, para fins de caracterização dos profissionais, questões sobre idade e sexo.

0 uso do instrumento adaptado e validado para o Brasil foi autorizado pelas autoras. A coleta de dados ocorreu entre dezembro de 2017 e janeiro de 2018, nos períodos matutino e vespertino, no ambiente de trabalho dos participantes. Os instrumentos foram respondidos, individualmente, em local privativo, com tempo médio de 30 minutos para preenchimento.

Os dados foram inseridos em planilha do $\mathrm{Mi}^{-}$ crosoft Excel com dupla conferência e analisados com auxílio do Software Statistical Package for the Social Sciences, versão 24.0. Realizou-se análise estatística descritiva, calculando a frequência absoluta e relativa para cada resposta dos itens avaliados. Consideraram-se positivas as respostas 'concordo totalmente/ concordo' ou 'frequentemente/sempre' para os itens formulados positivamente, e 'discordo totalmente/ discordo' ou 'nunca/raramente' para os itens formulados negativamente. As respostas 'uma ou duas vezes nos últimos 12 meses' e 'não aconteceu nos últimos 12 meses', relacionadas com os itens que avaliam frequência de situações no ambiente da Atenção Primária à Saúde, também, foram consideradas positivas, conforme o Medical Office Survey on Patient Safety Culture. Para as dimensões da cultura de segurança, foram calculados os percentuais de respostas posi- 
tivas, negativas e neutras. 0 percentual de resposta positiva de cada dimensão foi obtido por intermédio da média do percentual de respostas positivas dos itens que compõem a respectiva dimensão. Percentual igual ou acima de $75 \%$ foi considerado dimensão forte. Percentual igual ou abaixo de $50 \%$ foi considerado dimensão fraca/oportunidade de melhoria.

0 presente estudo seguiu a Resolução do Conselho Nacional de Saúde 466/12 e foi aprovado pelo Comitê de Ética em Pesquisa da Faculdade de Ciências da Saúde da Universidade de Brasília, Certificado de Apresentação de Apreciação Ética no 73448117.5.0000.0030, Parecer no 2.411.870/2017. Todos os participantes assinaram o Termo de Consentimento Livre e Esclarecido.

\section{Resultados}

Participaram do estudo 51 profissionais da Atenção Primária à Saúde, sendo 12 (23,5\%) técnicos de enfermagem, 11 (21,6\%) Agentes Comunitários de Saúde, oito $(15,7 \%)$ enfermeiros, sete $(13,7 \%)$ médicos, além de seis $(11,8 \%)$ profissionais de nível superior (odontólogo, terapeuta ocupacional, nutricionista, assistente social), cinco $(9,8 \%)$ profissionais de nível técnico (técnicos de higiene dental e técnicos administrativos) e dois $(3,9 \%)$ auxiliares operacionais de serviços de patologia clínica e laboratório. Profissionais do sexo feminino foram a maioria dos componentes, com $41(80,4 \%)$ participantes. A idade variou de 24 a 54 anos e média de 38,8 ( \pm 6,97 anos), predominando a faixa etária de 31 a 40 anos para 25 (49,0\%) profissionais. 0 tempo de trabalho no serviço variou entre menos de um ano e nove anos, média de 6,1 ( $\pm 2,8$ anos). A carga horária semanal de trabalho foi de 40 horas para todos os profissionais.

A avaliação dos percentuais de respostas positivas não revelou dimensões fortes da cultura de segurança no ambiente investigado. Como oportunidades de melhoria ou fragilidades do serviço, destacaram-se as dimensões relacionadas com o Ritmo e pressão no trabalho $(15,4 \%)$, Treinamento da equipe $(27,0 \%)$,
Processo de trabalho e padronização (33,0\%), Comunicação aberta (47,6\%), Comunicação sobre o erro $(40,8 \%)$, Apoio dos gestores na segurança do paciente $(40,2 \%)$ e com a Percepção geral da segurança do paciente e qualidade $(49,2 \%)$. A Tabela 1 apresenta o detalhamento do percentual de respostas positivas de cada dimensão relacionada com a cultura de segurança na Atenção Primária à Saúde.

Tabela 1 - Percepção dos profissionais da unidade de saúde quanto às dimensões da cultura de segurança do paciente de acordo com o Medical Office Survey on Patient Safety Culture. Brasília, DF, Brasil, 2018. (n=51)

\begin{tabular}{|c|c|c|c|c|}
\hline Dimensões & $\begin{array}{l}\text { Nega- } \\
\text { tivas }\end{array}$ & $\begin{array}{l}\text { Neu- } \\
\text { tras }\end{array}$ & $\begin{array}{l}\text { Posi- } \\
\text { tivas }\end{array}$ & $\begin{array}{l}\text { PRP* } \\
(\%)\end{array}$ \\
\hline Trabalho em equipe & 24 & 38 & 142 & 69,6 \\
\hline Ritmo e pressão no trabalho & 152 & 18 & 31 & $15,4^{+}$ \\
\hline Treinamento da equipe & 74 & 34 & 40 & $27,0^{+}$ \\
\hline Processo de trabalho e padronização & 88 & 44 & 65 & $33,0^{+}$ \\
\hline Comunicação aberta & 23 & 84 & 97 & $47,6^{+}$ \\
\hline Seguimento da assistência ao paciente & 33 & 36 & 112 & 61,9 \\
\hline Comunicação sobre o erro & 31 & 78 & 75 & $40,8^{\dagger}$ \\
\hline $\begin{array}{l}\text { Apoio dos gestores na segurança do pa- } \\
\text { ciente }\end{array}$ & 66 & 44 & 74 & $40,2^{\dagger}$ \\
\hline Aprendizagem organizacional & 33 & 33 & 80 & 54,8 \\
\hline $\begin{array}{l}\text { Percepção geral da segurança do paciente } \\
\text { e qualidade }\end{array}$ & 49 & 48 & 94 & $49,2^{+}$ \\
\hline
\end{tabular}

Tabela 2 apresenta a percepção dos profissionais quanto à qualidade da assistência, à segurança do paciente e à troca de informações na Atenção Primária à Saúde, evidenciando a frequência com que acontecem situações que podem expor pacientes a potenciais riscos.

A avaliação geral em segurança do paciente foi apontada como boa ou razoável para $80,4 \%$ dos profissionais como é apresentada na Tabela 3. Também se verificou predominância de avaliação boa/razoável das áreas de cuidado na Atenção Primária à Saúde. 
Tabela 2 - Frequência de situações ocorridas nos últimos 12 meses relacionadas com a segurança do paciente, qualidade e com a troca de informações. Brasília, DF, Brasil, 2018. (n=51)

\begin{tabular}{|c|c|c|c|c|}
\hline \multirow[t]{2}{*}{ Avaliação da segurança do paciente, qualidade do cuidado e troca de informações } & $\begin{array}{l}\text { Diaria- } \\
\text { mente }\end{array}$ & $\begin{array}{l}\text { Uma vez/ } \\
\text { semana }\end{array}$ & $\begin{array}{l}\text { Uma vez/ } \\
\text { mês }\end{array}$ & $\begin{array}{l}1,2 \text {, várias } \\
\text { vezes/ano* }\end{array}$ \\
\hline & n (\%) & n (\%) & n (\%) & n (\%) \\
\hline \multicolumn{5}{|l|}{ Problemas de segurança do paciente e qualidade da assistência } \\
\hline Um paciente não conseguiu uma consulta em até 48 horas para um problema sério/agudo & $1(2,4)$ & $6(14,6)$ & $2(4,9)$ & $32(78,1)$ \\
\hline No atendimento a um paciente foi utilizado prontuário/registro de outro paciente & - & - & - & $32(100,0)$ \\
\hline O prontuário/registro de um paciente não estava disponível quando necessário & $1(2,3)$ & $5(11,4)$ & $5(11,4)$ & $33(75,0)$ \\
\hline $\begin{array}{l}\text { Informações clínicas de um paciente foram arquivadas, digitalizadas ou inseridas no } \\
\text { prontuário/registro de outro paciente }\end{array}$ & - & - & $2(5,9)$ & $32(94,1)$ \\
\hline $\begin{array}{l}\text { Um equipamento necessário ao atendimento não funcionou adequadamente ou necessitava } \\
\text { de reparo ou substituição }\end{array}$ & $6(13,0)$ & $8(17,4)$ & $6(13,0)$ & $26(56,5)$ \\
\hline 0 paciente retornou à unidade de saúde para esclarecer ou corrigir uma prescrição & - & $3(8,1)$ & $9(24,3)$ & $25(67,6)$ \\
\hline $\begin{array}{l}\text { Os medicamentos utilizados por um paciente não foram revisados pelo profissional de } \\
\text { saúde durante sua consulta }\end{array}$ & - & - & $3(13,0)$ & $20(87,0)$ \\
\hline Os exames laboratoriais ou de imagem não foram realizados quando necessário & $13(31,0)$ & $1(2,4)$ & $5(11,9)$ & $23(54,8)$ \\
\hline $\begin{array}{l}\text { Os resultados de exames laboratoriais/de imagem não estavam disponíveis quando } \\
\text { necessário }\end{array}$ & $7(16,7)$ & $9(21,4)$ & $4(9,5)$ & $22(52,4)$ \\
\hline $\begin{array}{l}\text { Um resultado anormal de um exame laboratorial ou de imagem não foi acompanhado/ } \\
\text { avaliado em tempo hábil }\end{array}$ & - & $2(6,5)$ & $6(19,4)$ & $23(74,2)$ \\
\hline \multicolumn{5}{|l|}{$\begin{array}{l}\text { Problemas de troca de informações completas, precisas e pontuais em relação a } \\
\text { outros serviços }\end{array}$} \\
\hline Centros de imagem/laboratórios da Rede de Atenção à Saúde? & $11(33,3)$ & $7(21,2)$ & $2(6,1)$ & $13(39,4)$ \\
\hline Outros serviços de saúde/médicos da Rede de Atenção à Saúde? & $7(21,2)$ & $3(9,1)$ & $4(12,1)$ & $19(57,6)$ \\
\hline Farmácias? & $3(10,3)$ & $6(20,7)$ & $4(13,8)$ & $16(55,2)$ \\
\hline Hospitais? & $7(20,0)$ & $5(14,3)$ & $2(5,7)$ & $21(60,0)$ \\
\hline
\end{tabular}

*Respostas positivas, conforme o instrumento Medical Office Survey on Patient Safety Culture

Tabela 3 - Percepção dos profissionais da unidade de saúde quanto à avaliação global da qualidade do cuidado. Brasília, DF, Brasil, 2018. (n=51)

\begin{tabular}{|c|c|c|c|c|}
\hline \multirow{2}{*}{$\begin{array}{l}\text { Avaliação global da qualidade do cuidado e avaliação geral em relação à } \\
\text { segurança do paciente }\end{array}$} & Ruim & Razoável/Bom & Muito Bom & Excelente \\
\hline & n (\%) & n (\%) & n (\%) & n (\%) \\
\hline $\begin{array}{l}\text { Cuidado centrado no paciente: é sensível às preferências individuais, necessidades e } \\
\text { valores dos pacientes }\end{array}$ & $3(5,9)$ & $40(78,4)$ & $7(13,7)$ & $1(2,0)$ \\
\hline Cuidado efetivo: é baseado no conhecimento científico & - & $39(76,5)$ & $10(19,6)$ & $2(3,9)$ \\
\hline Cuidado pontual: minimiza esperas e atrasos potencialmente prejudiciais & $5(9,8)$ & $40(78,4)$ & $6(11,8)$ & - \\
\hline $\begin{array}{l}\text { Cuidado eficiente: garante um cuidado de bom custo-benefício (evita o desperdício, } \\
\text { uso excessivo e incorreto de serviços) }\end{array}$ & $2(3,9)$ & $41(80,4)$ & $8(15,7)$ & - \\
\hline $\begin{array}{l}\text { Cuidado imparcial: fornece a mesma qualidade de cuidados a todos os indivíduos, } \\
\text { independentemente de gênero, etnia, status socioeconômico, idioma, etc. }\end{array}$ & $2(3,9)$ & $31(60,7)$ & $11(21,6)$ & $7(13,7)$ \\
\hline $\begin{array}{l}\text { Avaliação geral em segurança do paciente: classificação dos sistemas e processos } \\
\text { clínicos que este serviço utiliza para prevenir, identificar e corrigir problemas que } \\
\text { tenham o potencial de afetar pacientes }\end{array}$ & $3(5,9)$ & $41(80,4)$ & $7(13,7)$ & - \\
\hline
\end{tabular}




\section{Discussão}

As limitações do estudo versam sobre o formato de coleta de dados que é dependente do interesse e disponibilidade do profissional de saúde em participar. Outras limitações estão relacionadas com a fonte de dados utilizada para avaliar a cultura de segurança que considerou o autorrelato do profissional envolvido, sem incorporar a análise de registros, observação da prática assistencial, percepção do paciente, dentre outros aspectos.

Apesar de refletir um diagnóstico situacional, o estudo traz contribuições para a prática profissional, levando a um processo de reflexão passível de modificação do ambiente organizacional fundamentada na tomada de decisão baseada em evidência. No contexto da ciência, os resultados do estudo, confrontados com a literatura nacional e internacional, reforçam que o processo de cultura de segurança é dinâmico, dependente da interação entre profissionais, seus pares, lideranças e paciente, assim como da interação entre esses atores com a estrutura do serviço e com o processo de trabalho. Essa realidade implica a necessidade de avaliações sistematizadas, a fim de, continuamente, levantar as necessidades de melhoria, rumo à obtenção de cuidados primários cada vez mais seguros.

Neste estudo, o trabalho em equipe, a aprendizagem organizacional e o seguimento da assistência do paciente receberam maiores pontuações. Esse resultado corrobora a literatura, quando aponta que os profissionais de saúde percebem um forte senso de trabalho em equipe dentro das unidades, relatam aprendizagem organizacional para melhoria dos processos de trabalho; no entanto, continuam identificando problemas de comunicação entre a equipe, respostas punitivas aos erros, problemas na frequência de relato de eventos e erros ao transferir pacientes para outros níveis de atenção à saúde ${ }^{(10)}$.

As dimensões da cultura de segurança refletem a maturidade organizacional em relação ao compromisso com o cuidado primário seguro. No Brasil, den- tre as dimensões melhores avaliadas, estão o trabalho em equipe e o acompanhamento do paciente ${ }^{(11-12)}$. No interior do estado de São Paulo, verificaram-se como fortalezas as dimensões trabalho em equipe e troca de informações com outras instituições, com 79\% de respostas positivas, acompanhamento do cuidado do paciente com $80 \%$ e segurança do paciente e problemas de qualidade com $83 \%$, enquanto que o suporte da liderança foi identificado como uma dimensão fraca com $47 \%{ }^{(11)}$. Entretanto, ainda, observam-se cenários em que prevalece a avaliação negativaa ${ }^{(12)}$, como ocorreu no presente estudo.

Diante disso, é necessária a busca de estratégias que auxilie o fortalecimento da cultura de segurança na prática da atenção primária, a ponto de modificar a forma de pensar o cuidado e promover a mudança atitudinal ${ }^{(1)}$. Intervenções educativas têm se mostrado efetivas para o aumento significativo do relato de incidentes $^{(13)}$. Nesse caso, portanto, o fomento de ações de vigilância em segurança do paciente deve ser um compromisso das lideranças, a fim de ampliar o relato de incidentes e qualificar as informações oferecidas.

Outras recomendações para melhorar esse cenário versam sobre a implantação de protocolos, qualificação profissional, melhoria da comunicação e resolutividade da assistência ${ }^{(12)}$. Destaca-se que a cultura de segurança do paciente pode apresentar diferença entre as categorias profissionais e, comumente, são mais baixas entre médicos e Agentes Comunitários de Saúde ${ }^{(14)}$. Dessa forma, a criação de um ambiente de aprendizagem organizacional, de modo a possibilitar a transferência de conhecimento relacionada com as boas práticas de segurança durante a prestação de cuidados é relevante para alavancar o cuidado primário seguro.

Neste estudo, participaram enfermeiros, médicos, Agentes Comunitários de Saúde, dentre outros profissionais com tempo de formação e experiência profissional diversificados. A literatura aponta que tempo de serviço ${ }^{(11-12)}$, trabalhar na Estratégia Saúde da Família ${ }^{(12)}$ e categoria profissional ${ }^{(11)}$ são fatores que estão significativamente relacionados com a 
percepção da cultura de segurança ${ }^{(12)}$. Desse modo, reconhecer os talentos existentes na organização e incentivar a aplicação do conhecimento de cada um em prol da melhoria dos processos de trabalho podem ser importantes ações no suporte organizacional à aprendizagem.

0 respeito entre colegas, autonomia profissional para aplicar novas ideias relacionadas com a segurança do paciente, valorização e incentivo dos chefes para uso e aplicação de novas habilidades, acrescidos da questão de entender que existem possíveis riscos e ser reconhecido ao aplicar novas habilidades relacionadas com a segurança do paciente no trabalho são ações percebidas como suporte organizacional e que favorecem a mudança do ambiente de prática ${ }^{(15)}$. Entretanto, ainda são observadas resistência às mudanças, excesso de trabalho na rotina do serviço, recebimento de críticas negativas diante da ocorrência de um erro e sentimento de ameaça ao aplicar novas habilidades. Tais fatores dificultam o processo de aprendizagem organizacional ${ }^{(15)}$ e impedem a evolução do processo de gestão da segurança.

0 acesso universal é um desafio importante para o Sistema Único de Saúde, devido ao aumento da demanda por ações e serviços, na medida em que a organização da rede, de forma regionalizada e hierarquizada, exige formulação e gestão de fluxos de cuida$\mathrm{do}^{(16)}$. Cabe salientar que a coordenação desses fluxos pode ser favorecida pelo uso de tecnologias digitais, tais como ferramentas de regulação e instrumentos de referência e contrarreferência que permitem a gestão dos processos e fluxos, além do acesso às informações de saúde do paciente em diferentes serviços ${ }^{(17)}$. Tal organização e coordenação contribuem para a segurança do paciente, uma vez que oportunizam o acesso à saúde, pois proporcionam a troca de informação entre os serviços, o acesso aos exames e aos seus resultados.

Apesar dos problemas levantados com o estudo, a avaliação geral da segurança foi apontada como positiva para os profissionais de saúde. Reconhecer os pontos positivos da instituição pode motivar o engajamento dos profissionais em busca de melhores práticas. Engajar profissionais nas ações de segurança do paciente mantém relação estatisticamente significativa com a cultura de segurança positiva e a redução de erros ${ }^{(18)}$. Importante destacar que a dinâmica da equipe influencia a coordenação do cuidado e as percepções da segurança do paciente, o que sugere que tornar a assistência mais segura implica prestar mais atenção em como se dá o trabalho colaborativo entre os profissionais de saúde ante a necessidade de coordenação do cuidado ${ }^{(19)}$.

A construção conjunta da cultura de segurança é dependente da compreensão de que o erro acontece devido a uma falha no sistema de saúde e a um processo de trabalho com barreiras ineficientes. Profissionais de saúde precisam ser corresponsáveis pelo diagnóstico de tais falhas e pelo planejamento de estratégias eficientes, devendo ser incluídos na gestão proativa dos riscos assistenciais e não somente na implementação prática das ações de segurança ${ }^{(20)}$. Portanto, os resultados mostram a necessidade de preparar profissionais de saúde, equipes de apoio e suas lideranças para gerenciar problemas relacionados com a assistência e engajar todos no processo de (re)pensar as práticas profissionais, a fim de fortalecer o cuidado primário seguro.

\section{Conclusão}

A avaliação da cultura de segurança do paciente na Atenção Primária à Saúde não identificou dimensões fortes. Verificaram-se problemas frequentes com o funcionamento de equipamentos necessários para o atendimento ao paciente, realização e disponibilidade dos resultados de exames laboratoriais ou de imagem. Constataram-se problemas relacionados com a troca de informações completas, precisas e pontuais com centros de imagem/laboratórios da Rede de Atenção à Saúde. Entretanto, a avaliação geral em relação à segurança do paciente e à avaliação global da qualidade do cuidado na Atenção Primária à Saúde foram apontadas como boas ou razoáveis. 


\section{Colaborações}

Bohrer JKL contribuiu para a concepção do projeto, análise e interpretação dos dados e redação do artigo. Vasconcelos ACL e Teixeira CC colaboraram para a análise e interpretação dos dados e redação do artigo. Bezerra ALQ, Andrade J e Santos PHF colaboraram para a análise e interpretação dos dados e revisão crítica relevante do conteúdo intelectual. Paranaguá TTB contribuiu para a concepção do projeto, análise e interpretação dos dados, redação do artigo e revisão crítica relevante do conteúdo intelectual. Todos os autores contribuíram para a aprovação final da versão a ser publicada.

\section{Referências}

1. World Health Organization (WHO). The third draft of the global patient safety action plan. [Internet]. 2021 [cited Mar 27, 2021]. Available from: https:// cdn.who.int/media/docs/default-source/patient-safety/gpsap/global-patient-safety-action-plan-2021-2030_third-draft_january-2021_ web.pdf?sfvrsn=6767dc05_15\&download=true

2. Lawati MHA, Dennis S, Short SD, Abdulhadi NN. Patient safety and safety culture in primary health care: a systematic review. BMC Fam Pract. 2018; 19(1):104. doi: https://doi.org/10.1186/s12875018-0793-7

3. Michel P, Brami J, Chanelière $M$, Kret $M$, Mosnier A, Dupie I, et al. Patient safety incidents are common in primary care: a national prospective active incident reporting survey. PLoS One. 2017; 12(2):e0165455. doi: https://doi.org/10.1371/ journal.pone.0165455

4. Cooper A, Edwards A, Williams H, Evans HP, Avery A, Hibbert P, et al. Sources of unsafe primary care for older adults: a mixed-methods analysis of patient safety incident reports. Age Ageing. 2017; 46(5):833-9. doi: https://doi.org/10.1093/ ageing/afx044

5. Auraaen A, Slawomirski L, Klazinga N. The economics of patient safety in primary and ambulatory care: flying blind. OECD Health Working Papers. 2018; 106:1-57. doi: https://doi.org/10.1787/baf425ad-en
6. Aguiar TL, Lima DS, Moreira MAB, Santos LF, Ferreira JMBB. Patient safety incidents in Primary Healthcare in Manaus, AM, Brazil. Interface. 2020; 24(Suppl 1):e190622. doi: https://doi. org/10.1590/interface.190622

7. Nora CRD, Beghetto MG. Patient safety challenges in primary health care: a scoping review. Rev Bras Enferm. 2020; 73(5):e20190209. doi: https://doi. org/10.1590/0034-7167-2019-0209

8. Andrade AM, Rodrigues JS, Lyra BM, Costa JS, Braz MNA, Dal Sasso MA, et al. Evolução do programa nacional de segurança do paciente: uma análise dos dados públicos disponibilizados pela Agência Nacional de Vigilância Sanitária. Visa Debate. 2020; 8(4):37-46. doi: https://doi. org/10.22239/2317-269x.01505

9. Timm M, Rodrigues MC. Cross-cultural adaptation of safety culture tool for Primary Health Care. Acta Paul Enferm. 2016; 29(1):26-37. doi: http:// dx.doi.org/10.1590/1982-0194201600005

10. Lawati MHA, Short SD, Abdulhadi NN, Panchatcharam SM, Dennis S. Assessment of patient safety culture in primary health care in Muscat, Oman: a questionnaire - based survey. BMC Fam Pract. 2019; 20(1):50. doi: https://doi.org/10.1186/ s12875-019-0937-4

11. Galhardi NM, Roseira CE, Orlandi FS, Figueiredo RM. Assessment of the patient safety culture in primary health care. Acta Paul Enferm. 2018; 31(4):409-16. doi: http://doi.org/10.1590/19820194201800057

12. Souza MM, Ongaro JD, Lanes TC, Andolhe R, Kolankiewicz ACB, Magnago TSBS. Patient safety culture in the Primary Health Care. Rev Bras Enferm. 2019; 72(1):27-34. doi: https://dx.doi. org/10.1590/0034-7167-2017-0647

13. González-Formoso C, Clavería A, FernándezDomínguez MJ, Lago-Deibe FL, Hermida-Rial L, Rial A, et al. Effectiveness of an educational intervention to improve the safety culture in primary care: a randomized trial. BMC Fam Pract. 2019; 20(1):15. doi: https://doi.org/10.1186/ s12875-018-0901-8

14. Raimondi DC, Bernal SCZ, Oliveira JLC, Matsuda LS. Patient safety culture in primary health care: analysis by professional categories. Rev Gaúcha Enferm. 2019; 40(esp):e20180133. doi: https:// doi.org/10.1590/1983-1447.2019.20180133 
15. Paranaguá TTB, Bezerra ALQ, Tobias GC, Ciosak SI. Support for learning in the perspective of patient safety in primary health care. Rev Latino-Am Enfermagem. 2016; 24:e2771. doi: http://dx.doi. org/10.1590/1518-8345.0784.2771

16. Silva LS, Menezes C, Nascimento LC, Nitscke RG, Duarte DC, Viegas SMF. Demanda espontânea e acesso no Sistema Único de Saúde: vivências de usuários da atenção primária. Av Enferm. 2021; 39(1):30-9. doi: http://doi.org/10.15446/ av.enferm.v39n1.85573

17. Ribeiro SP, Cavalcanti MLT. Primary health care and coordination of care: device to increase access and improve quality. Ciênc Saúde Coletiva. 2020; 25(5):1799-808. doi: https://doi. org/10.1590/1413-81232020255.34122019
18. Janes G, Mills T, Budworth L, Johnson J, Lawton R. The association between health care staff engagement and patient safety outcomes: a systematic review and meta-analysis. J Patient Saf. 2021; 17(3):207-16. doi: https://doi.org/10.1097/ PTS.0000000000000807

19. Blumenthal KJ, Chien AT, Singer SJ. Relationship among team dynamics, care coordination and perception of safety culture in primary care. Fam Pract. 2018; 35(6):718-23. doi: https://doi. org/10.1093/fampra/cmy029

20. Macedo LL, Haddad MCFL, Silva AMR, Girotto E. Culture of patient safety in primary health care in a large municipality in the perception of workers. Texto Contexto Enferm. 2020; 29:e20180410. doi:https://doi.org/10.1590/1980-265XTCE-2018-0410

\section{(cc) BY}

Este é um artigo de acesso aberto distribuído sob os termos da Licença Creative Commons 